\title{
Meta
}

Journal des traducteurs

Translators' Journal

\section{Delisle, J. et J. Woodsworth (dir.) (2007) : Les traducteurs dans l'histoire, Ottawa, Presses de l'Université d'Ottawa, 2 édition revue et corrigée, xxiii-393 p.}

\section{André Clas}

Volume 54, numéro 1, janvier 2009

URI : https://id.erudit.org/iderudit/029802ar

DOI : https://doi.org/10.7202/029802ar

Aller au sommaire du numéro

Éditeur(s)

Les Presses de l'Université de Montréal

ISSN

0026-0452 (imprimé)

1492-1421 (numérique)

Découvrir la revue

Citer ce compte rendu

Clas, A. (2009). Compte rendu de [Delisle, J. et J. Woodsworth (dir.) (2007) : Les traducteurs dans l'histoire, Ottawa, Presses de l'Université d'Ottawa, 2 édition revue et corrigée, xxiii-393 p.] Meta, 54(1), 174-176.

https://doi.org/10.7202/029802ar d'utilisation que vous pouvez consulter en ligne. 
Somme toute, même s'il y a peu d'apports nouveaux, l'ensemble est bien tenu, l'effort de cohésion et les notes de renvoi entres les articles sont appréciés, ces regards sur l'interprétation des langues de signes ont beaucoup d'intérêt. Nous ne regrettons qu'une seule chose, le coût élevé du volume qui le rend sûrement peu accessible en dehors des bibliothèques universitaires.

Danielle-Claude Bélanger

Université du Québec à Montréal, Montréal, Canada

\section{RÉFÉRENCES}

Giles, D. (1995): Basic Concepts and Models for Interpreter and Translator Training, Amsterdam/Philadelphia, John Benjamins.

Delisle, J. et J. Woodsworth (dir.) (2007): Les traducteurs dans l'histoire, Ottawa, Presses de l'Université d'Ottawa, $2^{e}$ édition revue et corrigée, xxiii-393 p.

Ce livre était à l'origine une gageure, mais il est heureux de voir aujourd'hui, une dizaine d'années plus tard, une deuxième édition, ainsi que quelques traductions dans diverses langues. Ce livre est en fait une esquisse - pouvait-on faire davantage en si peu de pages? C'est un condensé qui illustre les expériences antérieures et les résultats obtenus par des pionniers traducteurs. C'est en fait un livre de créations et de résultats que nous pouvons considérer comme prototypiques en ce sens qu'ils ne forment pas un tout mais seulement des exemples que l'on peut (doit?) multiplier et adapter en changeant de langue de référence ou de pays puisqu'il y aura beaucoup en commun mais également une constante individuelle à mettre en relief. Cela montre bien que l'ouvrage répond encore à un besoin et que l'entreprise est encore des plus méritoires. Rappelons-le, même si on le sait parfaitement, que résumer en si peu de pages l'histoire d'un certain nombre de traducteurs, et donc de la traduction, et par conséquent synthétiser l'ensemble de la civilisation mondiale des origines à nos jours, tant pour les sciences et la technologie que pour l'histoire, la littérature et les influences politiques et religieuses, est une entreprise sinon impossible du moins périlleuse, car il faut en peu de lignes dire l'essentiel et faire percevoir la trame de l'ensemble. Tout devient forcément élément de choix et bien évidemment interprétation et mise en relief de quelques aspects fondamentaux pour les orientations retenues. La critique devient aisée, car le choix des secteurs retenus peut être considéré comme partiel et surtout partial, et lié aux intérêts directs des auteurs. Mais il faut quand même souligner que l'essentiel pour ces secteurs est présent et que le choix n'est en quelque sorte qu'une mise en bouche qui laisse deviner l'attrait pour la suite et tout l'intérêt des développements qui s'annoncent et des compléments de lecture possibles. Les exemples donnés sont donc souvent à considérer comme des cas d'espèces hautement généralisables.

L'ouvrage suit un ordre logique et répartit la matière en neuf chapitres, chacun est suivi de références pour des lectures complémentaires. Ainsi au chapitre premier «Les traducteurs, inventeurs d'alphabets" font suite les chapitres où on les reconnaît comme «bâtisseurs de langues nationales, artisans de littératures, diffuseurs de connaissances, acteurs sur la scène du pouvoir, propagateurs de religions, importateurs de valeurs, consommateurs mais aussi compilateurs de dictionnaires terminologiques ». Le dernier chapitre s'attache au rôle des «interprètes témoins privilégiés de l'histoire». L'ouvrage se termine par deux annexes (légendes des illustrations; coauteurs), les «Références» et une «Bibliographie complémentaire - Domaines anglais et français (1995-2006)» et un «Index» des auteurs cités. 
Il y a, bien entendu, des choses qui manquent et qui peuvent paraître pour certains des oublis importants. Ainsi, dans le développement de la littérature, il faudrait souligner l'importance de Catherine II de Russie dans l'évolution de la culture russe car elle a créé, en 1762, une société pour la traduction des œuvres étrangères et, comme le souligne Hélène Carrère d'Encause (2002: 268) : «Le Cercle des traducteurs du corps d'infanterie noble, créé en 1762, accomplit un travail étonnant. Des dizaines de livres sont traduits à son initiative et mis rapidement sur le marché. Tous les genres sont représentés (abbé Prévost, Lesage, Fielding, Swift, contes de fées, récits, mythologies).» On voit donc que les traducteurs ont joué un grand rôle en Russie, puisqu'il y a là ce qu'on peut appeler une politique gouvernementale systématique de développement culturel par la traduction.

Même si la richesse bibliographique citée est très imposante et permet au lecteur de retrouver de nombreux compléments ou suppléments d'information, il y a bien évidemment des ouvrages oubliés et des références non citées parce qu'elles sont rédigées dans d'autres langues. Nous pensons notamment au travail de Claudia Wiotte-Franz (Hermeneus und Interpres, zum Dolmetscherwesen in der Antike, Saarbrücker Studien zur Achäologie und alten Geschichte, Band 16, 2001, 313 p.; cf. Meta 47-4, p. 639) qui est une prosopographie détaillée des interprètes du $\mathrm{VI}^{\mathrm{e}}$ siècle avant J.-C. au VI ${ }^{\mathrm{e}}$ siècle après J.-C.

En ce qui concerne les détails, il nous semble que le texte de la note 19 p. 63 est quelque peu ambigu puisqu'on lit: «Son origine (le yiddish) remonte au début du XII ${ }^{\mathrm{e}}$ siècle environ. Il s'est formé à partir d'un mélange de plusieurs dialectes germaniques parlés dans les ghettos d'Europe centrale. De là, il s'est réparti ailleurs en Europe.» Il nous semble que c'est plutôt l'inverse. Le yiddish appartient au moyen-haut-allemand, c'est en fait une variété du francique thiois et rhénan, qui se parlait déjà à l'époque de Charlemagne et certainement par Charlemagne lui-même, puisque c'était la langue de la région de Metz à Aix-la-Chapelle. La langue a, en même temps que certains locuteurs, "émigré» vers l'Europe centrale. Que le yiddish ait fait des emprunts à d'autres langues nous semble tout à fait normal, c'est le lot de toute langue, puisque aucune ne se suffit à elle-même pour désigner tout.

À la page 68 (Chapitre III), on peut lire: «Dans une métaphore qui transcende le temps et l'espace, Chaucer compare la traduction à l'action de labourer un ancien champ pour y refaire pousser du maïs.» Cela nous semble fort étrange puisque le mot maïs (maiz) date de 1519 et a été emprunté en 1493 au taino (langue des Arawaks, Grand Larousse encyclopédique). Il est donc originaire de l'Amérique, découverte 92 ans après la mort de Chaucer.

À la page 69, on trouve la phrase suivante: «En fait, les premiers États-nations ne sont apparus qu'à la Renaissance», affirmation qui nous semble quelque peu anachronique. Le mot État pour "désigner un groupement humain soumis à une même autorité" ne semble apparaître que vers 1500 et sans le sens moderne de "nation». Sans doute s'agit-il d'États nationaux, c'est-à-dire constitués de groupes humains, une ethnie, pour ne pas dire une tribu, ayant en commun un parler et soumis à un seigneur. Il est vrai qu'à ce moment, on n'est plus au VII ${ }^{\mathrm{e}}$ siècle où, par exemple, les Frisons refusèrent le christianisme des missionnaires Francs, mais acceptèrent la conversion des missionnaires venus d'Irlande et d'Angleterre, parce qu'ils étaient d'une tribu plus proche.

À la page 71, on lit: «Issu (Vondel) d'une famille d'immigrants du sud des Pays-Bas, il grandit dans une communauté baptiste d'Amsterdam.» Il faut présumer qu'il s'agit ici du terme «anabaptiste (1525) » puisque le sens n'est pas le même, le mot «baptiste» étant vraisemblablement un emprunt du XIX ${ }^{e}$ siècle. C'est en effet en 1535 qu'il y eut une répression des anabaptistes à Amsterdam et les groupements religieux se sont diversifiés. C'est ainsi que sont nés, à cette époque, les mennonites, du nom de leur fondateur Menno Simons, un ancien curé hollandais.

À la page 218, on peut trouver l'expression entre guillemets: «la pointe de l'iceberg» qui est, comme on le sait, le calque de "the tip of the iceberg». On peut se demander si les auteurs ont ainsi voulu illustrer le rôle des traducteurs dans l'enrichissement de la langue. 
À la page 227, l'exemple donné me semble quelque peu «court»: «Un seul exemple du passé: les Tchèques, entre le $\mathrm{XV}^{\mathrm{e}}$ et le $\mathrm{XVII}^{\mathrm{e}}$ siècle, ont traduit en latin leurs écrits, le plus souvent religieux et historiques, pour faire connaître leur "cause", c'est-à-dire pour justifier le mouvement hussite et ses conséquences.» On trouve dans tous les pays des "réformateurs». Au XIV siècle en Hollande, Geert Groote fonde, en réaction à la décadence du clergé, les «Frères de la vie commune», qui est un retour à la pure doctrine de l'Évangile, avec une vue sociale, mouvement qui connut un succès considérable, malgrél'hostilité des Dominicains et qui ne fut pas condamné par le concile de Constance. Érasme, traducteur célèbre, fut un de leurs élèves et également un précurseur de la Réforme et un critique acerbe de la corruption du clergé, comme le prouve son Éloge de la folie. La langue officielle, à l'époque, était, ne l'oublions pas, le latin et l'Église officielle toute-puissante.

Lors d'une troisième édition, il serait utile de corriger les coquilles de la page 10 (ajouté et non ajouter) et de la page 43 (quels et non qu'ils).

Pour terminer, on nous permettra de rappeler les numéros spéciaux de Meta sur la traduction biblique et religieuse, sur la traduction dans les divers pays ainsi que ceux sur l'histoire de la traduction (vol. 49-3, 50-4, 50-3), cf. <www.erudit.org/revue/META >.

André Clas

Université de Montréal, Montréal, Canada

Martin, R. (2004): Comprendre la linguistique, $2^{\mathrm{e}}$ édition, Paris, PUF, 206 p.

Comprendre la linguistique est un ouvrage qui servira de repère dans l'histoire de la linguistique. Il arrive à un moment où la discipline a relativement dépassé les hésitations des disciplines nouvelles et l'esprit conquérant affiché par les détenteurs des vérités vraies. Nous pouvons dire que cet ouvrage est fondé sur un bon diagnostic concernant l'évolution de la linguistique en tant que discipline: celle-ci a suffisamment mûri pour qu'une réflexion approfondie soit menée en vue d'en apprécier l'objet, les concepts, les outils méthodologiques et les résultats. Nous pensons que Robert Martin a vu juste; la preuve: le succès que cet ouvrage a eu auprès des linguistes. Une deuxième édition a vu le jour deux ans à peine après la parution de la première.

Cet ouvrage revendique dès le sous-titre, «épistémologie élémentaire d'une discipline », son terrain de réflexion. Pour l'auteur, il est clair que la discipline a besoin qu'on fasse le point pour poser les bonnes questions relatives aux domaines, aux méthodes et aux applications qu'on peut faire des travaux des linguistes. Aucune facette n'est omise: linguistique descriptive, linguistique théorique, linguistique générale, philosophie du langage, linguistique historique, linguistique appliquée, linguistique «stylistique». L'auteur aurait pu mener également sa réflexion sur la base des découpages reconnus au sein de la discipline: phonétique, phonologie, morphologie, syntaxe, sémantique, analyse du discours, etc.; il a préféré à cette approche celle qui situe la réflexion à un niveau beaucoup plus élevé, lui permettant ainsi d'englober ces derniers domaines dans sa présentation. Tous les domaines sont évoqués avec des illustrations suffisamment abondantes pour que le non-spécialiste saisisse les vrais enjeux de cette discipline.

Il serait difficile de rendre compte des mérites de cet ouvrage qui peut être considéré déjà comme un classique dans la matière. Nous nous contenterons de les ramener à trois: la limpidité de l'expression, la profondeur de la réflexion et la densité de la matière.

La limpidité de l'expression se vérifie sur plusieurs plans: le style, l'usage des termes techniques, le ton choisi pour mener une réflexion aussi profonde. Le style est dépouillé de toutes les lourdeurs propres aux textes de spécialité que leurs auteurs, par souci de clarté, surchargent le plus souvent d'explications et de toutes sortes de détails et de précisions qui 